PROCEEDINGS OF THE

AMERICAN MATHEMATICAL SOCIETY

Volume 125, Number 6, June 1997, Pages 1845-1851

S 0002-9939(97)04022-7

\title{
A NOTE ON THE CHERN-SIMONS INVARIANT OF HYPERBOLIC 3-MANIFOLDS
}

\author{
MINGQING OUYANG
}

(Communicated by Ronald Stern)

\begin{abstract}
In this note we study how the Chern-Simons invariant behaves when two hyperbolic 3-manifolds are glued together along incompressible thrice-punctured spheres. Such an operation produces many hyperbolic 3manifolds with different numbers of cusps sharing the same volume and the same Chern-Simons invariant. The results in this note, combined with those of Meyerhoff and Ruberman, give an algorithm for determining the unknown constant in Neumann's simplicial formula for the Chern-Simons invariant of hyperbolic 3-manifolds.
\end{abstract}

\section{INTRODUCTION}

Let $M_{1}$ and $M_{2}$ be oriented complete finite-volume hyperbolic 3-manifolds containing incompressible thrice-punctured spheres $S_{1}$ and $S_{2}$ respectively. Let $M_{i}^{\prime}=$ $M_{i}-N\left(S_{i}\right)$ where $N\left(S_{i}\right)$ is a neighborhood of $S_{i}$ in $M_{i}$. Denote by $S_{i}^{0}$ and $S_{i}^{1}$ the two copies of $S_{i}$ in $\partial M_{i}^{\prime}$. Let $\phi_{0}: S_{1}^{0} \rightarrow S_{2}^{0}$ and $\phi_{1}: S_{1}^{1} \rightarrow S_{2}^{1}$ be two homeomorphisms which are either both orientation-preserving or both orientation-reversing. Then one can glue $M_{1}$ and $M_{2}$ together to form a new 3-manifold $M$ by identifying $S_{1}^{0}$ and $S_{2}^{0}$ using $\phi_{0}$ and identifying $S_{1}^{1}$ and $S_{2}^{1}$ using $\phi_{1}$. As shown in [A1], $M$ is hyperbolic with volume equal to the sum of the volumes of $M_{1}$ and $M_{2}$.

Denote by $C S(M)$ the Chern-Simons invariant of a complete finite-volume hyperbolic 3-manifold $M$.

The main result of this note is the following

Theorem 1. If $M$ is obtained by gluing $M_{1}$ and $M_{2}$ together along copies of $S_{1}$ and $S_{2}$ via the same identification (i.e., $\phi_{0}=\phi_{1}$ ), then $C S(M)=C S\left(M_{1}\right)+C S\left(M_{2}\right)$.

In particular, let $L_{1}$ and $L_{2}$ be hyperbolic links in $S^{3}$ with projections as shown in Figure 1(a) and (b).

The hyperbolicity of $L_{1}$ and $L_{2}$ implies that the twice-punctured disks bounded by the trivial components are incompressible. By cutting $S^{3}-L_{1}$ and $S^{3}-L_{2}$ open and gluing together along the disks, one gets the complement of a hyperbolic link $L$ whose projection is shown in Figure 2.

Following [A1], we call $L$ a belted sum of $L_{1}$ and $L_{2}$. In this note we denote $L=L_{1} \oplus L_{2}(\bigcirc$ represents the trivial component).

Figure 3 shows that the Borromean rings form a belted sum of the Whitehead links of the opposite-handed clasps.

Received by the editors December 5, 1995.

1991 Mathematics Subject Classification. Primary 57N10; Secondary 57M25. 


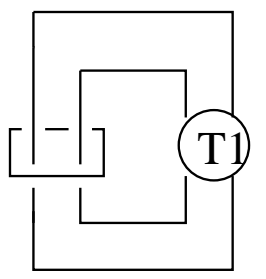

(a)

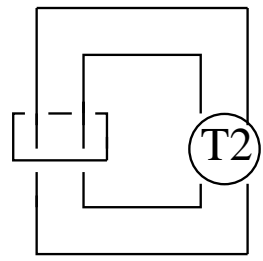

(b)

Figure 1

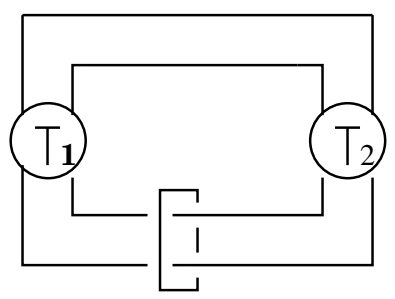

FigURE 2
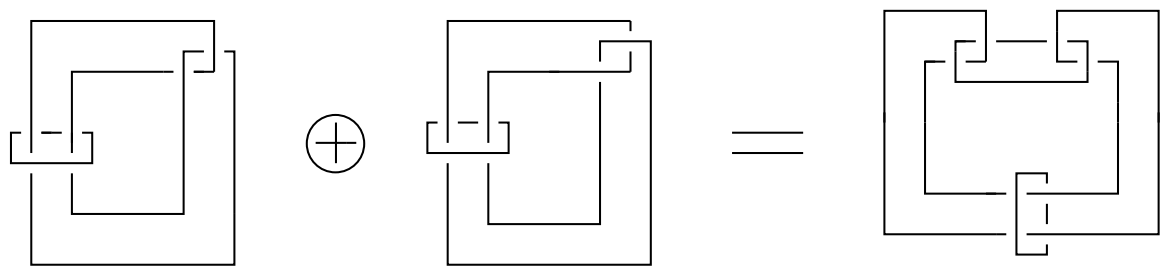

FiguRE 3

By the Mostow-Prasad Rigidity Theorem, $C S\left(S^{3}-L\right)$ is actually a link invariant of a hyperbolic knot or link $L$. One has the following

Corollary 2. $C S\left(S^{3}-L\right)=C S\left(S^{3}-L_{1}\right)+C S\left(S^{3}-L_{2}\right)$.

Example. As shown in [MR], the Chern-Simons invariant of the Whitehead link is $\pm 1 / 8$ depending on the handedness. Therefore the invariant for the Borromean rings is equal to 0 . This can also be seen from the amphicheirality of the Borromean rings $([\mathrm{MO}])$.

Unlike the volume, if a new manifold $M$ is obtained by gluing together $M_{1}$ and $M_{2}$ along copies of $S_{1}$ and $S_{2}$ via different identifications, then $C S(M) \neq$ $C S\left(M_{1}\right)+C S\left(M_{2}\right)$ in general. For example, denote by $\mu$ a mutation along either $S_{1}$ or $S_{2}$. If identifications $\phi$ and $\phi \circ \mu$ are used to glue $M_{1}$ and $M_{2}$ together to get $M$, then one would have $C S(M)=C S\left(M_{1}\right)+C S\left(M_{2}\right)+1 / 4$. This follows from the fact that a mutation along an incompressible thrice-punctured sphere changes the Chern-Simons invariant by $1 / 4$ ([MR]).

Given a hyperbolic knot or link $L$. Suppose that $L$ is in a particular regular alternating projection without any unnecessary crossings. Denote by $P$ the projection plane. Let $C_{1}, \cdots, C_{n}$ be $n$ nonisotopic embedded circles in the complement of 


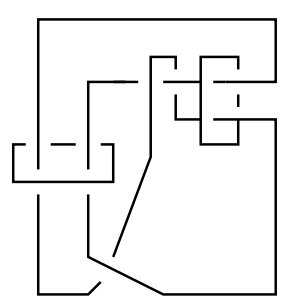

$\mathrm{L}$

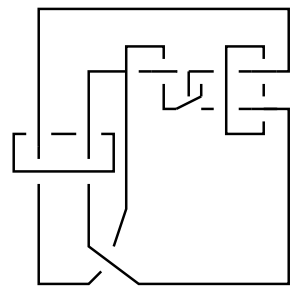

L'

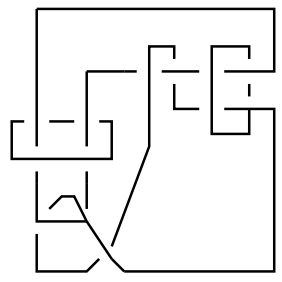

$\mathrm{L}^{\prime}$

Figure 4

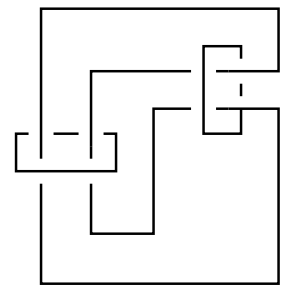

Borromean rings

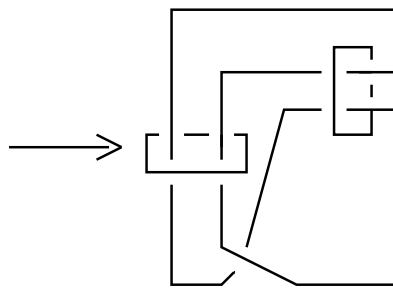

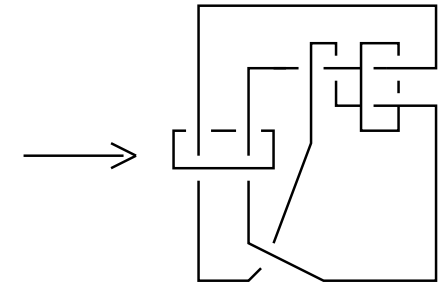

L

FIGURE 5

$L$ such that each $C_{i}$ intersects $P$ in exactly two points in different regions. Denote by $D_{i}$ the disk bounded by $C_{i}$ for $1 \leq i \leq n$. Assume further that $D_{i} \cap D_{j}=\emptyset$ for $i \neq j$ and each $D_{i}$ intersects $L$ in exactly two points. As shown in [A2], the new link $L^{\prime}=L \cup\left(\cup_{i=1}^{n} C_{i}\right)$, called an augmented alternating link, is also hyperbolic.

By performing different belt sums of two hyperbolic links, one may obtain different links whose complements have the same Chern-Simons invariant and the same volume. The following is an example.

In Figure $4, L$ is an augmented Whitehead link. $L^{\prime}$ and $L^{\prime \prime}$ are two belted sums of $L$ and the Whitehead link of right-handed clasp. Note that $L^{\prime}$ has three components while $L^{\prime \prime}$ has four. As shown in Figure 5, $L$ can be obtained from the Borromean rings by twisting one component a half twist and another component a full twist. The first operation changes the Chern-Simons invariant by $1 / 4$ ([MR]) and the second one does not change the homeomorphism type of the complement. Thus one has $C S\left(S^{3}-L\right)=1 / 4(\bmod 1 / 2)$. It follows from the corollary that $C S\left(S^{3}-L^{\prime}\right)=C S\left(S^{3}-L^{\prime \prime}\right)=1 / 4-1 / 8=1 / 8(\bmod 1 / 2)$.

Currently, there is a problematic aspect to calculating the Chern-Simons invariant of a hyperbolic 3-manifold in the "SnapPea" program of Weeks. The program is based on Neumann's simplicial formula $([\mathrm{N}])$ which involves an analytic function on hyperbolic Dehn surgery space that is only given up to an imaginary constant. Such a constant can be determined by a "bootstrapping" process, using surgery relations discovered among hyperbolic 3-manifolds. Theorem 1, together with the result of $[\mathrm{MR}]$ mentioned above, gives an algorithm to determine the constant for the hyperbolic 3-manifolds obtained by Dehn-surgeries on alternating hyperbolic knots and links. 


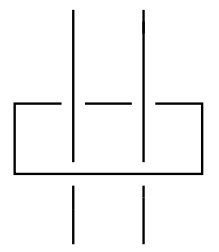

FiguRE 6
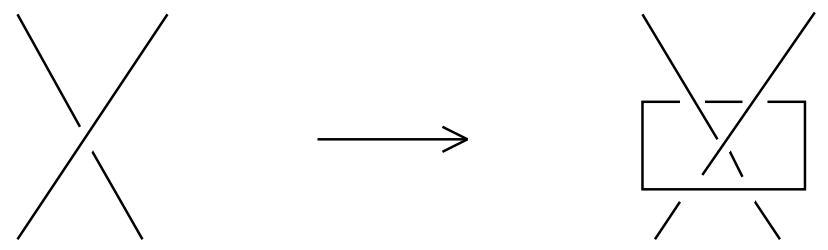

Figure 7

Recall that the simplicial formula for the Chern-Simons invariant $([\mathrm{N}])$ involves the same constant for the family of hyperbolic 3-manifolds obtained from a hyperbolic knot or link $L$ by Dehn surgeries (including $S^{3}-L$ ). Thus if one knows the Chern-Simons invariant of some manifold obtained from $L$ by a hyperbolic Dehn surgery, then one can determine the constant in the simplicial formula. We denote such a constant by $C_{L}$.

Suppose that $M$ is obtained from $L$ by a hyperbolic Dehn surgery and $C S(M)$ is not known. If $M$ is also the result of a hyperbolic Dehn surgery on another knot or link $L^{\prime}$ and one knows the Chern-Simons invariant of some manifold obtained from $L^{\prime}$ by a hyperbolic Dehn surgery, then one can calculate $C_{L^{\prime}}$ and hence $C S(M)$. This is how the bootstrapping process goes.

Definition. Let $L$ be a knot or link. Suppose $L$ is in a particular regular projection without any unnecessary crossings. If $L$ contains some portions as shown in Figure 6 , then delete the trivial component in each of these portions to obtain a new knot or link $L^{\prime}$; If $L$ does not contain such a portion, take $L^{\prime}=L$. The number of crossings in $L^{\prime}$ is called the bootstrapping complexity of $L$ with respect to the projection.

The following is the algorithm that allows one to determine the unknown constants for all alternating hyperbolic knots and links.

Given an alternating hyperbolic knot or link $L$. Let $n$ be the bootstrapping complexity of $L$ with respect to some projection which does not contain any unnecessary crossings. Let $L^{\prime}$ be a link obtained by adding a trivial component to $L$ around some crossing as shown in Figure 7.

Then $L^{\prime}$ is still hyperbolic ([A2]). Since $L$ can be recovered from $L^{\prime}$ by doing (1,0)-surgery along the added trivial component, $C_{L}$ can be determined if one knows $C S\left(S^{3}-L^{\prime}\right)$. If $L^{\prime}$ is a belt sum of two hyperbolic links, then one reduces the calculation for the Chern-Simons invariant to those for the two links with bootstrapping complexity less than $n$. Otherwise, by doing a half twist along the added trivial component in $L^{\prime}$, one can get rid of the crossing to obtain a new hyperbolic link $L^{\prime \prime}$ with bootstrapping complexity $n-1$. Following this process, one can 


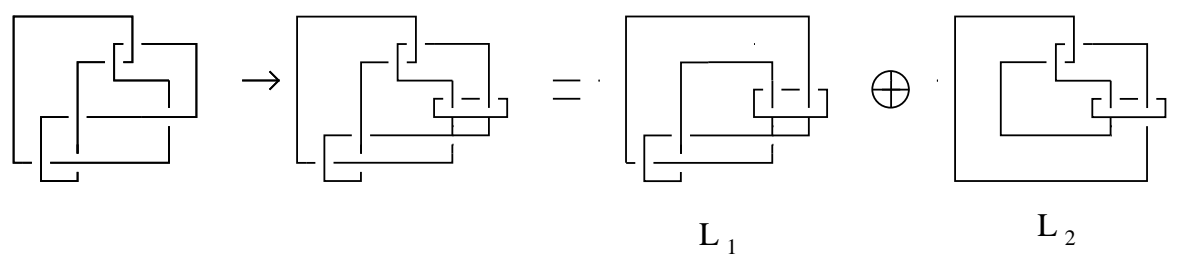

Figure 8

reduce bootstrapping complexity inductively to 0 . It is not hard to see that any knot or link of bootstrapping complexity 0 with respect to a particular projection is amphicheiral. As shown in [MO], the Chern-Simons invariant of an ampheichiral hyperbolic knot or link is equal to 0 .

Example. Figure 8 shows how the bootstrapping works for the knot $6_{3}$. Notice that $L_{1}$ is an augmented Figure- 8 knot and the Chern-Simons invariant of the Figure- 8 knot is 0 because of its amphicheirality.

\section{Proof of Theorem 1}

We first review the definition of the Chern-Simons invariant for a finite-volume cusped hyperbolic 3-manifold.

Let $M$ be an oriented complete hyperbolic 3-manifold of finite volume. Let $\mathcal{L}$ be a link in $M$. An orthonormal frame field $\alpha=\left(e_{1}, e_{2}, e_{3}\right)$ on $M-\mathcal{L}$ is said to have a special singularity at $\mathcal{L}$ if it has the following local structure near each component $K_{i}$ of $\mathcal{L}$ :

(a) vector $e_{1}$ is tangent to $K_{i}$ in the limit,

(b) vectors $e_{2}, e_{3}$ determine an index \pm 1 singularity in a small disk transverse to $K_{i}$.

A singular frame field $\alpha=\left(e_{1}, e_{2}, e_{3}\right)$ on $M$ is called linear if in every cusp the $e_{3}$-vectors are perpendicular to the horospheres and point outwards, and the $e_{1^{-}}$, $e_{2}$-vector fields are parallel on each horosphere.

Denote by $\omega_{i j}$ the Riemannian connection form associated to the hyperbolic metric on $M$ defined on the orthonormal frame bundle $F(M)$ of $M$. As in [Y], for every simple closed curve $C$ in $M$ and an orthonormal frame field $\beta$ defined on a tubular neighborhood of $C$ whose first component is tangent to $C$, the torsion of $C$ with respect to $\beta$ is defined by

$$
\tau(C, \beta)=-\int_{s_{\beta}(C)} \omega_{23}
$$

where $: s_{\beta}: C \rightarrow F(M)$ is the section defined by $\beta$.

If $\beta^{\prime}$ is another such frame field, then as shown in $[\mathrm{Y}]$,

$$
\tau\left(C, \beta^{\prime}\right)-\tau(C, \beta) \in 2 \pi \mathbf{Z} .
$$

Thus one has a well-defined torsion $\tau(C)$ modulo $2 \pi$.

Let $\alpha$ be a linear singular frame field on $M$ having a special singularity at some link $\mathcal{L}$. Denote by $Q$ the Chern-Simons 3-form defined on $F(M)$. In $[\mathrm{M}]$, the 
Chern-Simons invariant of $M$ is defined by

$$
C S(M)=\frac{1}{8 \pi^{2}} \int_{s_{\alpha}(M-\mathcal{L})} Q-\frac{1}{4 \pi} \sum_{i=1}^{n} \tau\left(K_{i}\right) \quad\left(\bmod \frac{1}{2}\right)
$$

where $s_{\alpha}: M-\mathcal{L} \rightarrow F(M)$ is the section defined by $\alpha$ and $K_{i}(1 \leq i \leq n)$ are the components of $\mathcal{L}$.

The important fact is that $C S(M)$ does not depend on the choice of $(\mathcal{L}, \alpha)$.

To prove the theorem, let $S_{1}$ and $S_{2}$ be two incompressible thrice-punctured spheres properly embedded in $M_{1}$ and $M_{2}$ respectively. Let $M_{i}^{\prime}=M_{i}-N\left(S_{i}\right)$ where $N\left(S_{i}\right)$ is a neighborhood of $S_{i}$ in $M_{i}$. Denote by $S_{i}^{0}$ and $S_{i}^{1}$ the two copies of $S_{i}$ in $\partial M_{i}^{\prime}$. Suppose that $M$ is obtained from $M_{1}$ and $M_{2}$ by gluing $S_{1}^{0}$ to $S_{2}^{0}$ and $S_{1}^{1}$ to $S_{2}^{1}$ using the same homeomorphism $\phi$.

Let $\alpha_{1}$ be a linear singular frame field on $M_{1}$ which has a special singularity at some link $\mathcal{L}_{1} \subset M_{1}$. Denote $S_{1} \cap \mathcal{L}_{1}=\left\{p_{1}, \cdots, p_{n}\right\}$. Then $\alpha_{1}$ restricts to a frame field on $S_{1}-\left\{p_{1}, \cdots, p_{n}\right\}$. The identification $\phi$ induces a frame field on $S_{2}-\left\{\phi\left(p_{1}\right), \cdots, \phi\left(p_{n}\right)\right\}$.

As remarked in $[\mathrm{M}]$, if one replaces a singular frame field used in the definition of $C S(M)$ by a so-called homotopically linear one at the cusps, then (1) still holds. Such a frame field merely requires that $e_{1}$ and $e_{2}$ be homotopic to parallel vector fields on each horosphere.

Using this remark, one can first extend the frame field on $S_{2}-\left\{\phi\left(p_{1}\right), \cdots, \phi\left(p_{n}\right)\right\}$ to the cusps of $M_{2}$ so that it is homotopically linear there.

Choose $n$ simple closed curves $\gamma_{1}, \cdots, \gamma_{n}$ in $M_{2}$ such that each $\gamma_{i}$ intersects $S_{2}$ precisely in $\phi\left(p_{i}\right)$. The obstruction to extending the frame field to the rest of $M_{2}$ is represented by a link $\mathcal{L}_{2}^{\prime}$ in the complement of $S_{2} \cup\left(\cup_{i=1}^{n} \gamma_{i}\right)$. As in [M], one thus obtains a singular frame field $\alpha_{2}$ having a special singularity at $\mathcal{L}_{2}=\mathcal{L}_{2}^{\prime} \cup\left(\cup_{i=1}^{n} \gamma_{i}\right)$.

Since $S_{1}$ (resp. $\left.S_{2}\right)$ does not separate $M_{1}$ (resp. $\left.M_{2}\right), \mathcal{L}_{1} \cup\left(\cup_{i=1}^{n} \gamma_{i}\right)$ forms a new link $\mathcal{L}_{1}^{\prime}$ in $M$. The singular frame fields $\alpha_{1}$ and $\alpha_{2}$ are glued together along copies of $S_{1}$ and $S_{2}$ via the same identification $\phi$ to form a singular frame field $\alpha$ on $M$ having a special singularity at $\mathcal{L}=\mathcal{L}_{1}^{\prime} \cup \mathcal{L}_{2}^{\prime}$. Also, to define $\tau\left(K_{i}\right)$ for each $K_{i} \subseteq \mathcal{L}_{1} \subset M_{1}$ one needs an orthonormal frame field $\beta_{i}$ on a neighborhood of each such $K_{i}$. Since the same identification $\phi$ is used to glue along copies of $S_{1}$ and $S_{2}$, one can simply choose an orthonormal frame field on a neighborhood of each $\gamma_{i}$ such that its first component is tangent to $\gamma_{i}$ and the second and the third component are constant along $\gamma_{i}$ and agree with those of $\beta_{i}$ on the copies of $S_{1}$ and $S_{2}$. Thus one obtains a frame field needed to define the torsion of each component of $\mathcal{L}_{1}^{\prime}$ in $M$. It follows that

$$
\begin{aligned}
C S(M) & =\frac{1}{8 \pi^{2}} \int_{s_{\alpha}(M-\mathcal{L})} Q-\frac{1}{4 \pi} \sum_{K_{i} \subset \mathcal{L}} \tau\left(K_{i}\right) \\
& =\frac{1}{8 \pi^{2}} \int_{s_{\alpha}(M-\mathcal{L})} Q-\frac{1}{4 \pi} \sum_{K_{i} \subset \mathcal{L}_{1}^{\prime}} \tau\left(K_{i}\right)-\frac{1}{4 \pi} \sum_{K_{i} \subset \mathcal{L}_{2}^{\prime}} \tau\left(K_{i}\right) \\
& =\frac{1}{8 \pi^{2}} \int_{s_{\alpha}(M-\mathcal{L})} Q-\frac{1}{4 \pi} \sum_{K_{i} \subset \mathcal{L}_{1}} \tau\left(K_{i}\right)-\frac{1}{4 \pi} \sum_{i=1}^{n} \tau\left(\gamma_{i}\right)-\frac{1}{4 \pi} \sum_{K_{i} \subset \mathcal{L}_{2}^{\prime}} \tau\left(K_{i}\right)
\end{aligned}
$$




$$
\begin{aligned}
& =\frac{1}{8 \pi^{2}} \int_{s_{\alpha_{1}\left(M_{1}-\mathcal{L}_{1}\right)}} Q-\frac{1}{4 \pi} \sum_{K_{i} \subset \mathcal{L}_{1}} \tau\left(K_{i}\right) \\
& \quad+\frac{1}{8 \pi^{2}} \int_{s_{\alpha_{2}}\left(M_{2}-\mathcal{L}_{2}\right)} Q-\frac{1}{4 \pi} \sum_{K_{i} \subset \mathcal{L}_{2}} \tau\left(K_{i}\right) \\
& =C S\left(M_{1}\right)+C S\left(M_{2}\right) .
\end{aligned}
$$

\section{ACKNOWLEDGEMENTS}

The author would like to thank the referee for many helpful comments and suggestions.

\section{REFERENCES}

[A1] C. Adams, Thrice-punctured spheres in hyperbolic 3-manifolds, Trans. Amer. Math. Soc. 287 (1985), 645-656. MR 86k:57008

[A2] C. Adams, Augmented alternating link complements are hyperbolic, London Math. Soc. Lecture Notes 112 (D. B. A. Epstein, ed.), 115-130. MR 89f:57003

[M] R. Meyerhoff, Density of the Chern-Simons invariant for hyperbolic 3-manifolds, in Lowdimensional topology and Kleinian groups, London Math. Soc. Lect. Notes 112, D. B. A. Epstein, editor, Cambridge University Press, (1987), 217-240. MR 88k:57033a

[MO] R. Meyerhoff and M. Ouyang, The $\eta$-invariant of cusped hyperbolic 3-manifolds, to appear in Canadian Math. Bull.

[MR] R. Meyerhoff and D. Ruberman, Mutation and the $\eta$-invariant, J. Differential Geom. 31 (1990), 101-130. MR 91j:57017

[N] W. Neumann, Combinatorics of triangulations and the Chern-Simons invariant for hyperbolic 3-manifolds, in Topology'90, Proceedings of the Research Semester on Low Dimensional Topology, de Gruyter Verlag, 1992. MR 93i:57020

[Y] T. Yoshida, The $\eta$-invariant of hyperbolic 3-manifolds, Invent. Math. 81 (1985), 473-514. MR 87f:58153

Department of Mathematics, University of Michigan, Ann Arbor, Michigan 48109

E-mail address: mouyang@math.lsa.umich.edu 